\title{
Die Pianistin spricht. Überlegungen zur Epistemologie von Vertonungsanalysen und ihrer Funktion in musikwissenschaftlicher Forschung
}

\author{
ANNEGRET HUBER, WIEN
}

An der Prämisse, dass eine Pianistin wie Clara Wieck/Schumann ${ }^{1}$ in ihren Gedichtvertonungen ,spricht', ist grundsätzlich nichts auszusetzen. Daraus ergibt sich jedoch im Hinblick auf die Analyse ihrer Gedichtvertonungen eine Reihe epistemologischer Fragen, die in diesem Beitrag diskutiert werden sollen. Dabei wird zunächst explizit unterschieden zwischen der Untersuchung des, Technischen' der kompositorischen Praktik Clara Wieck/Schumanns (das unter Umständen Rückschlüsse auf das implizite Wissen der Pianistin und Liedbegleiterin zulässt) zum einen und dem Sozialen ihrer damit verbundenen diskursiven Praxis zum anderen. Es wird nicht zuletzt auch um die Auslotung eines Graubereichs gehen, in dem die Analytikerin in Worten über die Kompositionsweise der Pianistin spricht, die sich ja nur in Tönen äußerte. Ins Zentrum rückt so die epistemische Funktion des analysierenden Subjekts, an dessen Aussagen zu unterscheiden ist, inwiefern diese über eigene Wahrnehmungen und ihre Praktiken des Lesens, Hörens, Analysierens etc. oder über Eigenschaften der Gedichtvertonung der Komponistin gemacht werden.

Wenn wir nun die Formulierung „Die Pianistin spricht" in jenem ganz wörtlichen Sinn² verstehen wollen, der kommunikative Aspekte ihrer kompositorischen Tätigkeit betonen soll, treten zur Frage, wie sie denn komponierend spreche, auch jene nach den Adressat*innen ihrer Äußerungen sowie die nach ihrer Person als Sprechende in den Fokus. Die künstlerische Praktik der Vertonung eines Gedichts wird dabei nicht selten sprachlich unsauber und epistemologisch undifferenziert in eins gesetzt zu sozialen Praxen der Mitteilung. Aus soziologischer Perspektive besteht hier jedoch ein wichtiger Unterschied: Ist an Praktiken allein die Art ihrer Ausführung zu untersuchen, sind an Praxen ihre soziale Situiertheit und ihre Formation durch Praktiken von Interesse. Abzugrenzen sind wiederum Praktiken und Praxen von Handlungen, bei denen die Motivationen von Handelnden (etwa in Form von Interviews) als Sinnstiftungszentrum erforscht

1 Obwohl der hier erörterte historische Zeitraum einer ist, in dem die Komponistin/Pianistin schon ihren Ehenamen Schumann führte, wird hier über sie als Wieck/Schumann gesprochen, um sie von ihrem Ehemann Schumann zu unterscheiden und zu vermeiden, dass sie als "Clara" wie die weniger ernst zu nehmende Persönlichkeit wirkt.

2 Wenn man die Formulierung im Titel auch als Metapher interpretieren wollte, könnten noch die Fragen nach der Medialität ihres kompositorischen, Sprechens' und der Aussagekraft der dabei verwendeten Zeichen thematisiert werden. Diese beiden Aspekte werden in diesem Beitrag nicht eigens theoretisch problematisiert. Dennoch liegen entsprechende Differenzierungen dem hier praktizierten Analyseansatz zugrunde. 
werden sollen ${ }^{3}$. Aus den fachgeschichtlich jüngeren praxeologischen Theorien ergeben sich im Vergleich mit den durch sie abgelösten Handlungstheorien neue Differenzierungsmöglichkeiten: „Von Handlungstheorien aus betrachtet, sind Praktiken im Wesentlichen Handlungen ohne ,Akteure', nämlich ein Strom des Handelns ohne Stilisierung eines souveränen Zentrums und rationalen Ursprungs. Praktiken sind primär eine neue, Individuen dezentrierende Art, das Handeln zu konzipieren." ${ }^{4}$ Dabei ergibt sich "eine Praxis als Vollzugswirklichkeit, die sich aus der Verkettung von Einzelpraktiken als Ereignisse bildet. Dies zwingt dazu, die variablen Bedingungen des Vollzugs der Praxis situationsanalytisch zu identifizieren, also das Zusammenkommen und -wirken von sozialisierten Körpern mit materialen Artefakten und Dingen sowie mit diskursiven und symbolischen Formationen zu untersuchen. ${ }^{55}$ Inwiefern eine Gedichtvertonung als materiales Artefakt untersucht werden kann, muss an dieser Stelle nicht diskutiert werden, denn die "grundsätzliche Annahme, ein Ding und damit auch ein Artefakt müsse aus Materie geformt sein [...] schließt vieles in der Welt vom Ding-Sein aus und ist daher nicht unumstritten." ${ }^{16}$ Es existieren vielmehr auch Ansätze, die die "Materialität als Vorbedingung des ArtefaktSeins aufgegeben [haben], und es stellt sich die Frage, ob Artefakte [...] Entitäten eigener Ordnungind, die sich allein durch ihr menschbedingtes Werden definieren. ${ }^{17}$

Durch die Anwendung dieser drei soziologischen Kategorien sollen also in diesem Beitrag zwei Momente lokalisierbar werden: zum einen die kompositorische Praktik der Komponistin - typischerweise der Anwendungsbereich strukturanalytischer Methodik -, die deutlicher von der Sozialität ihres künstlerischen Tuns abgegrenzt und ebenso wie die Komposition als solche von der Person der Komponistin unterschieden werden soll, sowie zum anderen die Praktik des Musikanalysierens, ihr Übergehen von strukturanalytischen Argumentationen in ihre kompositionsgeschichtlichen oder musikbiographischen Kontextualisierungen und die epistemische Funktion von Analysierenden. Es werden hier also zwei Analysen vorgestellt: die (Struktur-)Analyse einer Gedichtvertonung von Clara Schumann vor dem Hintergrund der praxeologischen Bedingungen ihrer Entstehung und eine Meta-Analyse eben dieser Analyse. Dabei soll jeweils im ,Dreischritt' die Frage nach kompositorischen Praktiken, Praktikenketten und Handlungen verfolgt werden. Der Meta-Analyse wird eine eigene Vertonungsanalyse zugrunde gelegt, um

3 Stefan Hirschauer, "Praktiken und ihre Körper. Über materielle Partizipanden des Tuns”, in: Doing culture. Neue Positionen zum Verhältnis von Kultur und sozialer Praxis, hrsg. von Karl H. Hörning et al., Bielefeld 2004, S. 73-91, hier S. 73.

4 Stefan Hirschauer, „Verhalten, Handeln, Interagieren zu den mikrosoziologischen Grundlagen der Praxistheorie”, in: Praxistheorie. Ein soziologisches Forschungsprogramm, hrsg. von Hilmar Schäfer, Bielefeld 2016, S.45-67, hier S. 46.

5 Frank Hillebrandt, „Vergangene Praktiken. Wege zu ihrer Identifikation”, in: Praktiken der Frühen Neuzeit: Akteure, Handlungen, Artefakte, hrsg. von Arndt Brendecke (= Frühneuzeit-Impulse, Bd. 3), Köln/Weimar/Wien 2015, S. 34-45, hier S. 40.

6 Christina Tsouparopoulou und Thomas Meier, "Artefakt”, in: Materiale Textkulturen. Konzepte - Materialien Praktiken, hrsg. von Thomas Meier et al. (= Materiale Textkulturen, Bd. 1), Berlin/München/Boston 2015, S. 47-62, hier S. 51.

7 Ebd., S. 52. 
beispielhaft einige strukturanalytische Konventionen herbeiführen und diskutieren zu können und gleichzeitig den Vorwurf der Wadlbeißerei zu vermeiden.

\section{A) Spricht die Komponistin als Pianistin?}

\section{Praktik des Komponierens}

intertexturell

Für die Musikanalyse wurde mit der Vertonung von Friedrich Rückerts „Die gute Nacht, die ich dir sage" WoO 17 bewusst eine schlicht gehaltene ausgewählt, um die unausgesprochene Prämisse, dass eine Pianistin in ihren Kompositionen am virtuosen Klaviersatz zu erkennen sei, nicht zum Zirkelschluss werden zu lassen. Wenn aber an diesem Beispiel Komponieren als Praktik untersucht werden soll, muss vorab festgehalten werden, dass dies nicht so umstandslos durch eine Strukturanalyse geleistet werden kann, denn eine Komposition ist nicht die (ihrem Wesen nach prozesshafte) Praktik als solche, sondern lediglich das Ergebnis von dieser. Eine Analyse kann also nicht mehr als ein Versuch der Rekonstruktion einer Praktik werden - wenngleich dadurch analytisch herausgearbeitet werden kann, dass die Komponistin sichtlich Entscheidungen getroffen hat, um eine Komposition in ihrer Eigenart hervorzubringen. Welche (Beweis-)Funktion dabei die Komposition als solche im Forschungssetting und in zweiter Instanz die strukturanalytisch erbrachten Befunde im musikhistorischen Narrativ besitzen könnten, wird zu diskutieren sein. Die folgende Strukturanalyse ist also weder umfassend noch vollständig, weil es unmöglich ist, aus dem zeitlichen Verlauf der Komposition auf die Chronologie des Kompositionsprozesses zu schließen; es werden vielmehr jene Aspekte abgehandelt, an deren Beispiel Aussagequalitäten und Argumentationsweisen veranschaulicht und erörtert werden können.

Was findet also die Komponistin im Gedicht vor, das ihr Anlass für kompositorische Entscheidungen bietet? Rückerts Gedicht hat eine recht originelle Strophenform, die erst im 18. Jahrhundert entstanden und im 19. Jahrhundert schon wieder in den Hintergrund getreten war: Bemerkenswert am Vierzeiler ist die sehr unterschiedliche Länge der 1./3. bzw. 2./4. Verse einer Strophe: Charakteristisch ist an daran der „überraschende Wechsel zwischen dem neunsilbigen Vers und dem kurzen Viersilber" ${ }^{\prime \prime}$, der noch dadurch verstärkt wird, dass die längere Zeile zusätzlich in einer unbetonten Endung nachklingt und die anschließende kürzere mit einer Betonung endet und so geradezu abrupt stoppt.

8 Horst Joachim Frank, Handbuch der deutschen Strophenformen, München/Wien 1980, S. 129. 
XXIII.

Die gute Nacht, die ich dir sage,
Freund, hörest du;
Ein Engel, der die Botschaft trage,
Geht ab und zu.
Er bringt sie dir, und hat mir wieder
Den Gruß gebracht:
Dir sagen auch des Freundes Lieder
Nun gute Nacht.

Abbildung 1: Friedrich Rückerts Gedichtvorlage ${ }^{9}$ nebst entsprechender Abstraktion der Strophenform ${ }^{10}$

Durch Kreuzreime entstehen enge strophische Beziehungen zwischen den Verspaaren. Inhaltlich ist diese Strophenform mit "freundlicher, tändelnder Munterkeit"11 konnotiert, wenngleich später das Spiel mit Unerwartetem auch zum Austragen von "seelische[n] Spannungen und zur Bezeichnung wahrgenommener Widersprüchlichkeiten"12 herangezogen wurde. An Rückerts Realisierung dieser Form im vertonten Gedicht könnten die beiden Enjambements in der zweiten Strophe mit der Frage interessant werden, ob und wie Clara Schumann ihnen Rechnung trägt oder nicht.

Ausgangspunkt für die Vertonung ist also (neben seinem poetischen Inhalt) die artifiziell gestaltete Sprache des Gedichts mit seiner Schall- und ,Augen'-Form. Der Rhythmus der gebundenen Sprache kommt dadurch in ein zu bestimmendes Verhältnis mit dem Musikrhythmus. Hätte die Komponistin diesen im ersten Verspaar ganz der Metrik des Gedichts untergeordnet, wäre eine Realisierung wie im linken Beispiel naheliegend gewesen.
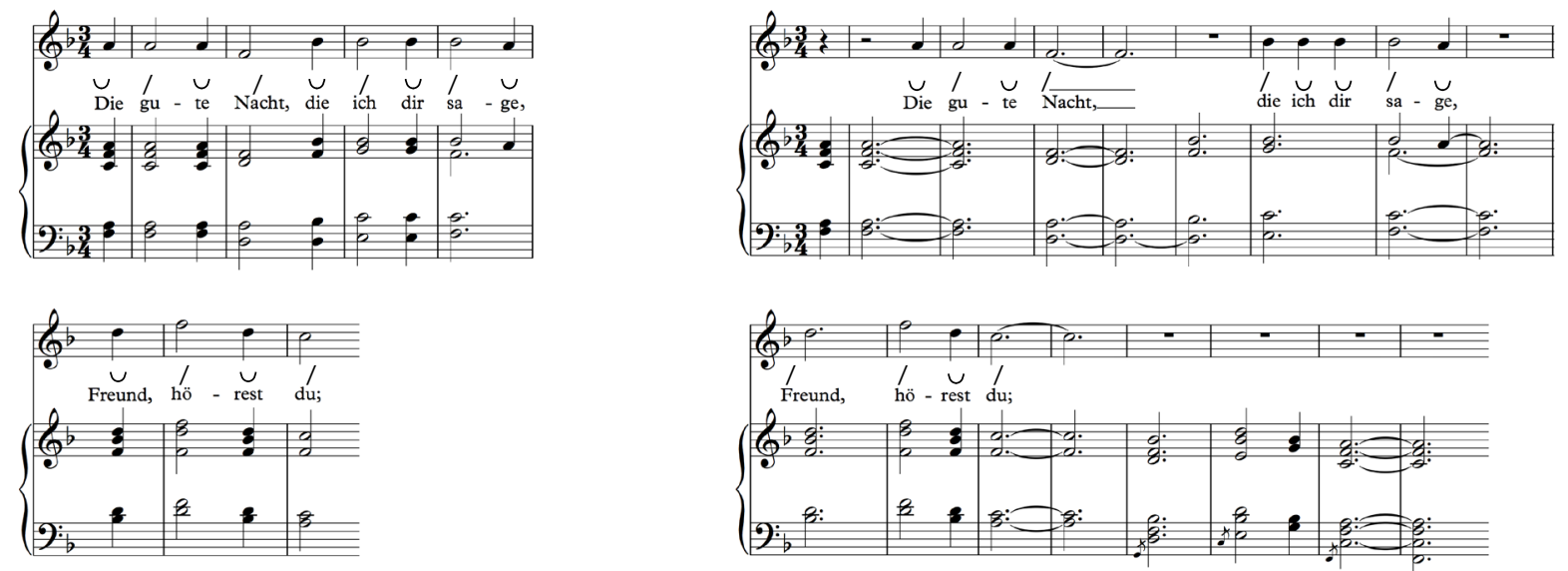

Abbildung 2: Musikrhythmus und Versmetrik übereinstimmend (links). Bei Clara Schumann: Musik überformt Versmetrik (rechts).

9 Friedrich Rückert, Gesammelte Gedichte, Bd. 1, Erlangen 1834, hier S. 205.

10 Frank, Handbuch der deutschen Strophenformen, S. 129.

11 Ebd.

12 Ebd. 
Die Komponistin entschied sich jedoch für eine andere Lösung: Die ganztaktigen Pausen in der Singstimme vollziehen die Interpunktion des Satzes nach und weniger die Versgliederung des Gedichts. Zwei Silben, die im Gedicht eigentlich unbetont sind (die vierte im ersten Vers und die erste im zweiten) werden in der Vertonung betont. Dadurch tritt die Anrufung des "Freundes" im zweiten Vers hervor. Warum statt des eigentlich betonten "[die] ich [dir sage]" (fünfte Silbe des ersten Verses) dem Relativpronomen „die [ich dir sage]" auf Zählzeit 1 des sechsten Taktes untergeordnet wird, bedarf eines Kommentars: Nach dem zwei Takte lang klingenden "Nacht" (Takt 3/4) und der anschließenden ganztaktigen Pause (Takt 5) wirkt die nicht-auftaktige Fortsetzung in Takt 6 etwas eilend - so als ob der Stillstand durch die Bewegung der drei Viertel in Takt 6 überwunden werden muss. Darüber hinaus verlängert das textfreie melodische Echo in der Oberstimme des Klaviers (Takt 13-16) den Inhaltsbereich dieses Doppelverses. Die nachstehende Darstellung lässt auch sehen, dass dieses Mittel der Gewichtungsumkehrung (Rechtecke in gelb und grün) weitere Worte hervortreten lässt: Neben dem bereits erwähnten "Freund" in Takt 9 sind dies "Dir" in Takt 39 und "Jetzt" in Takt 43 (Trapeze in grün und blau). Mögliche Gründe dafür ergeben sich aus dem Gedichtinhalt.

Im Schaubild ist jeweils ein Doppelvers in einer Musikzeile wiedergegeben; dadurch kann nachvollzogen werden, wie der Zeilenwechsel zwischen zwei Verspaaren des Gedichts in seiner Vertonung berücksichtigt werden kann - oder auch nicht: Die doppelt gestrichelte violette Linie markiert im Gedicht vorgesehene Orte des Zeilenwechsels. Vor Takt 9, 21 und 43 korreliert jeweils eine Pause mit dem Zeilenwechsel; die kurzen, jeweils viersilbigen Verse sind im Anschluss daran mit einem fast identischen melodischen Profil ausgestattet. Ausnahmen werden durch die blauen Dreiecke hervorgehoben. In den Zeilen 1, 2 und 4 könnte der Eindruck entstehen, dass die Reimstruktur des Gedichts nachvollzogen wird.

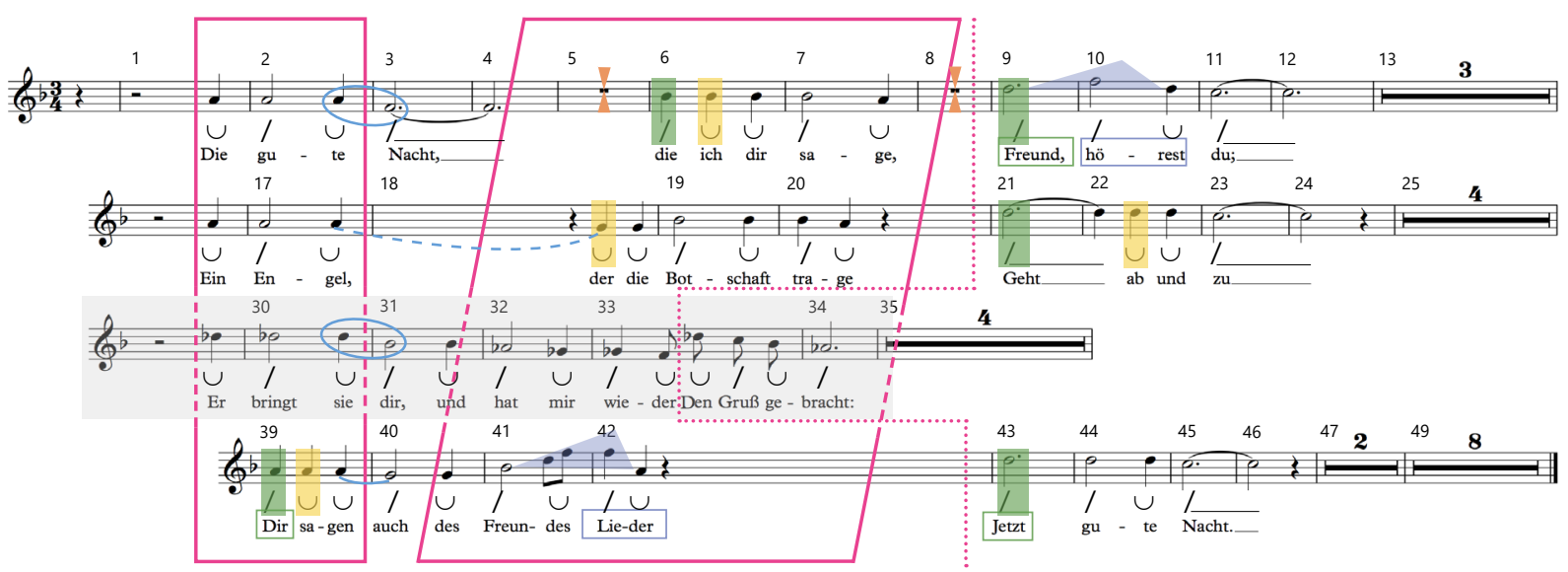

Abbildung 3: Visualisierung der Überformung der Strukturen des Gedichts durch seine Vertonung 
Ganz anders hingegen Zeile 3: Hier wird in Takt 33 der Zeilenwechsel mitten im Takt überspielt, was wieder durch den Inhalt des Gedichts an dieser Stelle begründet werden kann. Dies ist auch vor dem Hintergrund zu sehen, dass sowohl für diesen Abschnitt eine recht weit von der Grundtonart (F-Dur) entfernte Tonart (Des-Dur) gewählt wurde als auch im Klaviersatz ein deutlicher Strukturwechsel zu beobachten ist (grau unterlegt). Wenn man nun argumentieren wollte, dass der in Takt 33 unberücksichtigte Zeilenwechsel das Enjambement des Verspaars abbildet, müsste man sich gleichzeitig darüber wundern, dass ihm gleich darauf in Takt 42 nicht Rechnung getragen wird.

Des Weiteren ist zu beobachten, dass das neunsilbige Maß der ungeradzahligen Verse in musikalischer Ausarbeitung durchaus unterschiedlich lang gestaltet wird: Die Halbverse rücken sukzessive enger zusammen (vgl. in Pink das Parallelogramm vs. Rechteck); dies korreliert direkt mit der (gelegentlich auch fehlenden) Interpunktion durch Kommata im entsprechenden Vers. Die ,Gelenkintervalle' Terz und Sekund (türkis markiert) folgen wiederum in ihrer Anordnung der Strophenstruktur. Insgesamt wird die 2x2-teilige Gedichtform kompositorisch durch eine (im Wesentlichen) dreiteilige Anlage überformt. Hinzu treten musikformale Erweiterungen ,ohne Worte' - und zwar nicht nur (wie oben schon erwähnt) durch das Echo der Singstimme im Klavier (Takt 13-16), sondern auch durch den Wechsel von Begleitmustern und Zwischenspielen. Ein achttaktiges Nachspiel, das sogar noch Neues ins Spiel bringt, geht noch weiter über die Gedichtform hinaus. Wodurch es motiviert sein könnte, ist an dieser Stelle des strukturanalytischen Prozesses noch nicht zu beurteilen. Bis hierher wurde punktuell lediglich das Verhältnis zwischen Texturen der artifiziell gestalteten Sprache und ihrer Vertonung bestimmt. Erst im nächsten Schritt werden Relationen untersucht, die in den Vordergrund treten, wenn sowohl Gedicht als auch Vertonung als Text betrachtet werden.

\section{Exkurs: Strukturanalytische Aussagequalitäten}

Zur Erfassung intertextureller Beziehungen konnten die strukturanalytisch begründeten Aussagen recht einfach sein: Sie bezogen sich auf ziemlich offensichtliche Sachverhalte und beanspruchten nur wenig Raum für Interpretationen; Antworten auf Fragen, die größeren Begründungsaufwand nach sich ziehen könnten, wurden noch vermieden (wie etwa die nach der Bedeutsamkeit des Nachspiels). Häufig waren konstative Aussagen im Sinne Austins mög-

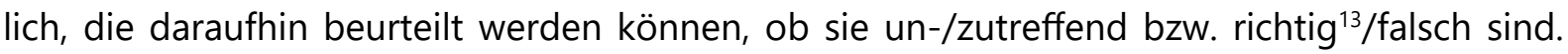
Die vorangegangenen analytischen Befunde sollten also ohne großen Widerspruch akzeptiert werden können.

Dennoch werden gelegentlich Einwände erhoben, wenn etwa ein Widerspruch zwischen Versakzent und Wortakzent (wie in der Vertonung realisiert) erkannt wird, weil das jambische

13 Von der Gegenüberstellung wahr/falsch wird hier im Gefolge des späten Nelson Goodman abgesehen, der Wahrheit als Kriterium für Aussagen über Kunst verwirft und stattdessen deren Richtigkeit betont. 
Versmaß als allzu rigide empfunden wird. Im hier analysierten Beispiel könnte dies in den geradzahligen Versen (beginnend in den Takten 9, 21 und 43) der Auftakt des zweihebig jambischen Verses sein, der durch die schwebende Betonung ${ }^{14}$ der Vertonung zum Volltakt wird und dadurch ,irgendwie' plausibler wirkt, wenn dadurch Worte - der Prosa gleich - ,natürlicher' hervorgehoben werden. Aber eben die Strenge des Versmaßes gehörte zu den Forderungen im Zuge der einflussreichen Opitz'schen Versreform, auch wenn später in der Heine-Zeit das ,Zusammenklappen' von Wortakzent und Versakzent zunehmend als ästhetisch unbefriedigend erachtet wurde, sodass mit der Ambiguität der schwebenden Betonung reizvollere Strukturen realisiert wurden - gerade so, wie Clara Schumann ihre Vertonung gestaltete. Solche sehr berechtigten Einsprüche aber verweisen auf ein gewisses Missverhältnis, wenn (gar nicht so selten) analytische Befunde mit der Attitüde und für die Aussagequalität konstativer Sprechakte ungeeigneten Formulierungen vorgetragen werden, ohne dass jene Kontexte nachgewiesen werden, durch die sie als Konstativa überhaupt möglich werden könnten. Dieses Beispiel markiert damit auch den Übergang von konstativen Sprachakten zu anderen Aussagequalitäten.

\section{intertextuell}

Mit der Frage nach der Intertextualität tritt auch die Bedeutung von Texten (oder Textähnlichem) in den Fokus. Dadurch ändert sich auch die Qualität der Aussagen: Während über Merkmale von Texturen einfachere und punktuelle Aussagen möglich sind, werden Texte erst zu solchen, wenn die Strukturen, aus denen sie sich zusammensetzen, als syntaktische Folge erkannt und durch den Leseakt mit Sinn erfüllt werden. Die Begründung der Ergebnisse von Leseakten zieht jedoch größeren Argumentationsbedarf nach sich - und das ganz besonders, wenn sich ein ,Text' (wie Musik) nicht aus bedeutungstragenden, sondern gewissermaßen bedeutungsoffenen Zeichen zusammensetzt.

Clara Wieck/Schumann wählte in dieser Vertonung eine effiziente Methode zur Semantisierung von Musikstrukturen, indem sie die Viertonfolge, zu der in Takt 33/34 (siehe Abbildung 4) die Worte "den Gruß gebracht" gesungen worden waren, im Zwischenspiel des Klaviers abspaltet, sodass dieser motivisch überbrachte ,Gruß' gewissermaßen das Wiedereinsetzen der Singstimme zu den Worten "Dir sagen auch des Freundes Lieder" motiviert.

Hier erhalten Musikzeichen ihre Bedeutung also durch den vertonten Text, was einfachere Argumentationen erlaubt, als wenn die Komponistin versucht hätte, Musikzeichen ,ohne Worte' so zu gestalten, dass sie über Musik hinausgehende Inhalte imitierten oder symbolisierten (Stichworte: Tonmalerei und Leitmotiv). Kompositorische Kontexte ergeben sich hier lediglich aus musik- wie literaturtheoretischen Hintergründen ihrer Zeit; andere Annahmen inhärenter

14 Als schwebende Betonung wird die ausgleichende Akzentuierung von Verspartien in Widerspruch zum regulären Versmaß bezeichnet, wobei Hebung und Senkung aneinander angeglichen werden. (Vgl. in Eichendorffs Mondnacht folgende Verse: "Und meine Seele spannte T Weit ihre Flügel aus”. Aus dem regulären $\cup / \cup / \cup / \cup \mid \cup / \cup / \cup /$ wird dabei $\cup / \cup / \cup / \cup \mid / / \cup / \cup /$.) 
Bedeutung, die erst durch entsprechende Analysen an Musik herangetragen werden (wie etwa die, dass die Bedeutung von Musikzeichen in ihnen selbst liegt und diese sich für Codierungen oder Chiffrierungen eignen), müssen hier nicht bemüht werden.

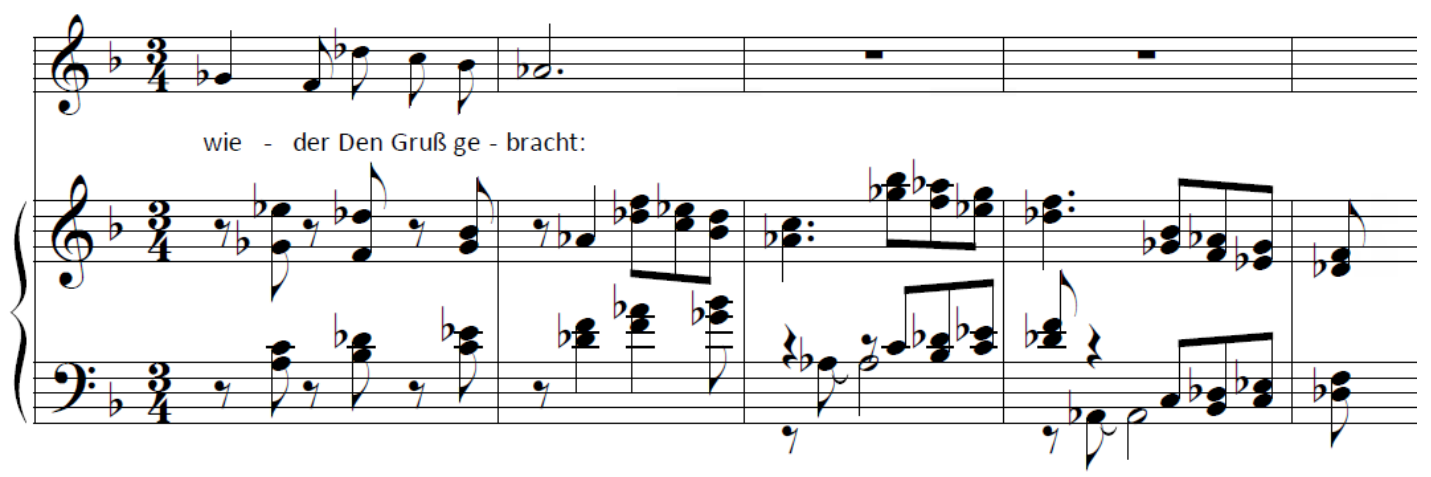

Abbildung 4: Takt 33-37: Semantisierung eines Motivs im Klavier durch Juxtaposition des vertonten Texts

Bemerkenswertes im Klavier als anzunehmender Ausdrucksebene der komponierenden Pianistin

Die bisherigen analytischen Befunde verweisen noch nicht notwendigerweise auf eine Komponistin mit besonderer Expertise für das Klavier. Wer jedoch schon einmal im Tonsatzunterricht angewiesen wurde, enge Akkordschichtungen in den unteren Registern bis zur Mittellage des Klaviers tunlichst zu vermeiden und möglichst keine Terzen zu verdoppeln, wird sich über Akkordschichtungen wie diese in Takt 18 wundern:

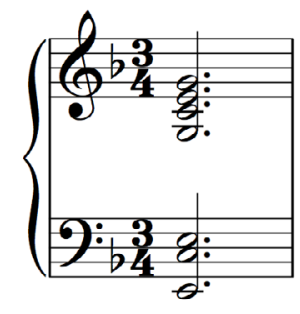

Abbildung 5: Takt 18: enge Akkordschichtung in der Mittellage des Klaviers mit drei verdoppelten Terzen 
Wenn man aber an anderen Stellen der Vertonung sieht, dass, terzenbehaftete' Strukturen in Verbindung mit einer speziellen Stimmführung nicht nur zu einer speziellen Charakteristik des Klavierklangs führt, ...

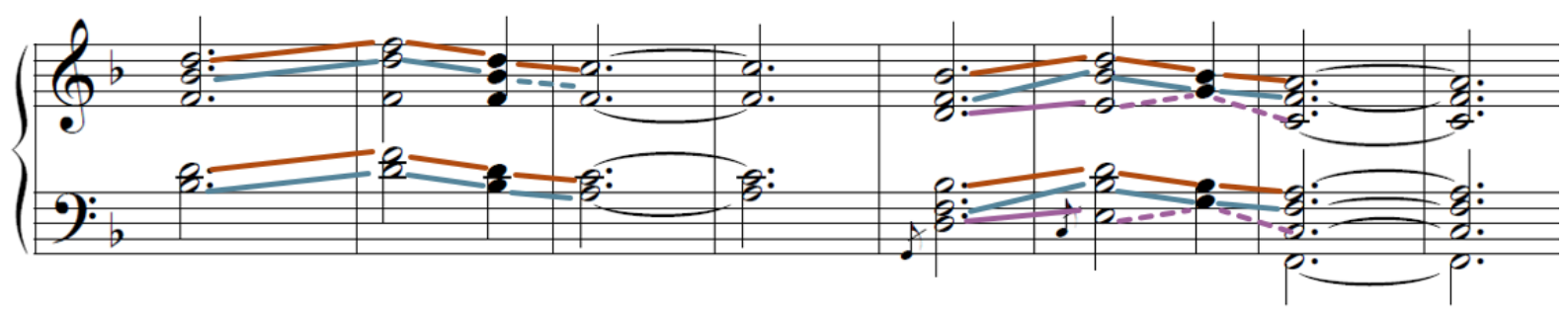

Abbildung 6: Takt 9-16: Austerzung/Parallelführung der Hände

... sondern auch beobachten kann, dass harmonisch kaum zu deutende Vierklänge (wie hier $e$-g-as-c in Takt 26) als chromatische Terzverschiebung auf dem Weg vom f-Moll-Dreiklang zum dominantischen Vierklang des Terzquartakkords erklärt werden können ...

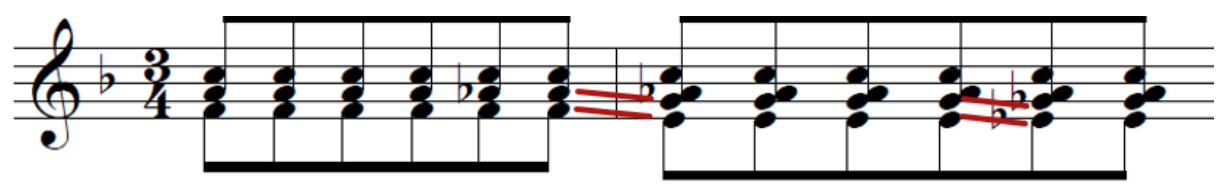

Abbildung 7: Takt 25/26: Stimmführung (Terzenverschiebung) zur Erklärung von Zusammenklängen im homophonen Satz

... und hier sogar clusterartige Stimmkreuzungen durch Stimmführungsgeschehen schlüssig argumentiert werden können, ...

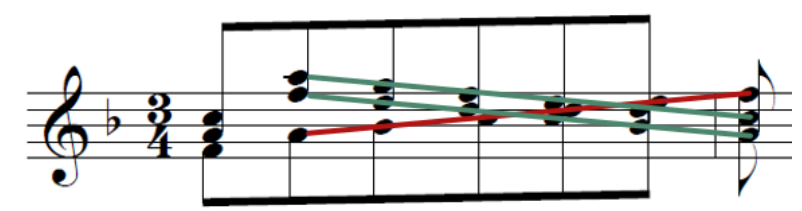

Abbildung 8: Zusammenklänge nur durch Stimmführung erklärbar

... dann könnte das die Annahme nahelegen, dass sich auch das $c^{1}$ in der Mitte des engen Akkords aus der melodischen Verlängerung des vorangegangenen Stimmverlaufs ergibt.

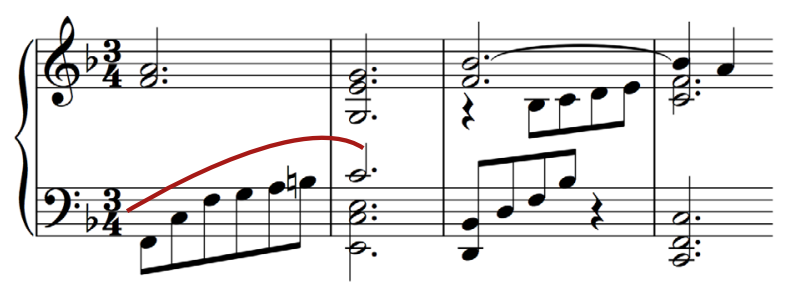

Abbildung 9: Takt 17/18 - analytisch begründete Umschrift 
Bemerkenswerterweise findet sich ein Beleg für diese Hypothese in einem Autograph: ${ }^{15}$ Die Achtel der linken Hand sind teilweise nach oben und nach unten gehalst, sodass die obere Halsung eine gewisse Zweistimmigkeit suggeriert, bei der die nach oben gehalste Stimme genau dem Stimmverlauf entspricht, der zum $c^{1}$ in der Mitte des Akkords führt.

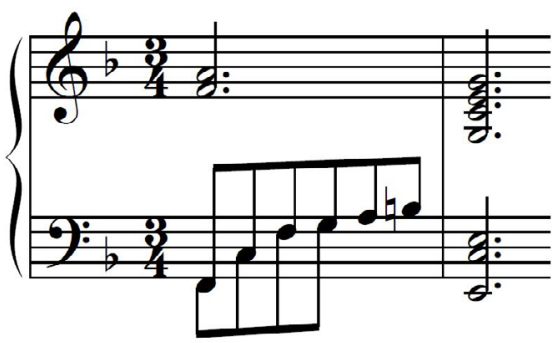

Abbildung 10: Takt 17/18 Schreibweise nach dem Berliner Autograph

Dies könnte die Vermutung nahelegen, dass auch das Nachspiel ebenfalls als zwei melodische Hauptstimmen zu denken sein könnte. Ungeachtet der Umstände der Entstehung der Komposition - Clara Wieck/Schumann hatte die Vertonung ihrem Mann zum Geburtstag geschenkt - werden diese jedoch als nichts Anderes als zwei Stimmen im musikalischen Satz aufgefasst und nicht als Verkörperungen der Stimmen zweier Menschen.

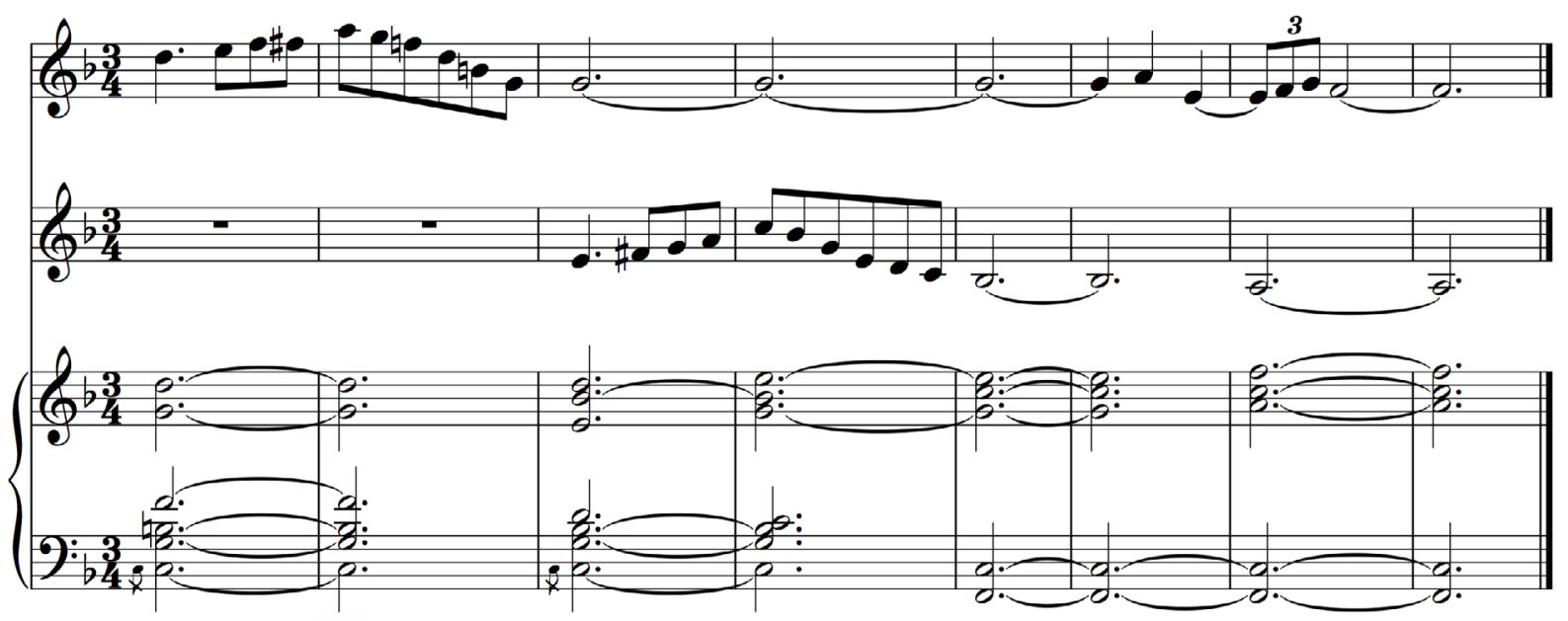

Abbildung 11: Nachspiel mit zwei hypothetischen Hauptstimmen (analytisch begründete Umschrift)

15 Bei diesem Autograph handelt es sich nicht um das Widmungsautograph, das Clara Wieck/Schumann ihrem Mann 1841 zum Geburtstag geschenkt hatte, sondern um ein anderes im Bestand der Staatsbibliothek zu Berlin - Preußischer Kulturbesitz, in dem alle 23 Lieder der Komponistin enthalten sind (D-B Mus.ms.autogr. Schumann, K. 5 (6)). Das hier analysierte Lied wurde aber de facto erst 1990 aus dem Nachlass veröffentlicht (Clara Schumann, Sämtliche Lieder, hrsg. von Joachim Draheim et al., Bd. 2: Unveröffentlichte Lieder, Wiesbaden 1990). 
Rückschlüsse aus der Vertonungsanalyse zur kompositorischen Praktik

Durch die Strukturanalyse konnte die Pianistin trotz der recht sparsamen Interpretationen der Befunde ein wenig in ihrer Komposition zum Sprechen gebracht werden. Ihre Entscheidungen als Elemente der Praktik einer Komponistin zeigen etwas, das als spezielles Wissen einer Pianistin gelesen werden kann: Die Komponistin spricht auch dann als Pianistin, wenn sie sich nicht als Virtuosin zeigt; sie scheint ansatzweise aus Notationsweisen zu sprechen, die vermuten lassen, dass sie damit auf die Möglichkeiten des Klangaufbaus auf einem Flügel ihrer Zeit verweist und auf ihr eigenes pianistisches Können, eine entsprechende (nicht ganz einfache) Klanggestalt auch wirklich zu erzeugen. Allerdings ist hier ebenso zu beobachten, wie sich hypothetische Aussagen über die Praktiken der Komponistin mit denen über die Wahrnehmungen und Sinnzuschreibungen der Analytikerin vermischen.

\section{Praxisformation um Clara Schumanns kompositorische Praktik}

Clara Wieck/Schumanns Praktik des Komponierens ist eingebettet in eine Kette von Praktiken, die nicht allein von ihr selbst durchgeführt wurden; sie münden in eine Praxisformation, die situationsanalytisch untersucht werden kann. Ein Ansatz, soziologische Situationsanalysen auch auf historische Gegenstände anzuwenden, stammt von Adele E. Clarke ${ }^{16}$; die von ihr vorgeschlagenen ,Mapping'-Techniken erfassen nicht nur alle Elemente der untersuchten historischen Praktiken, sondern ebenso jene heutiger und früherer Forschungspraktiken. ${ }^{17}$ Bei diesem Verfahren ${ }^{18}$ erfasst man zunächst in einer "messy map" "alle materiellen, symbolischen, menschlichen und nicht-menschlichen Elemente [...], die in der spezifischen Situation eine Rolle spielen und [...] dabei so dargestellt werden, wie sie von den Betroffenen in der Situation (also u. a. den Erforschten) und dem Analytiker [sic] selbst definiert werden." ${ }^{19}$ Solche "Definitionen' sind anfangs noch eher Hypothesen, die vom/von der Analytiker/in stets evaluiert und weiter differenziert werden können, je nachdem, welche Elemente sich in seinem Forschungsprozess als mehr oder weniger relevant erweisen. Eine "ordered situational map" in Form einer Tabelle (s. u.) gibt Aufschluss darüber, nach welchen Elementen überhaupt gesucht werden muss. Ausgehend von den noch assoziativen Anordnungen der Elemente auf "messy maps" können „relational maps" entworfen werden, indem die Beziehungen eines Elements zu jedem

16 Adele E. Clarke, Carrie E. Friese und Rachel S. Washburn, Situational Analysis: Grounded Theory After the Interpretive Turn, Thousand Oaks, CA, ${ }^{2} 2018$.

17 Vgl. Clarke/Friese/Washburn, Situational Analysis, Kapitel 13 „Mapping Historical Discourse Materials".

18 Eine konzise und gleichzeitig aussagekräftige Übersicht findet sich hier: „Ein Weg zur Repräsentativität nach dem ,postmodern turn'? - Adele E. Clarkes Ansatz der Situationsanalyse", auf: Uni Freiburg, <https://institut.so ziologie.uni-freiburg.de/2015ws-repraesentatitvitaet-qualitativer-forschung/wpg_9406.html> (23.09.2020). Sie bezieht sich allerdings noch auf die Erstausgabe, in der Adele Clarke ihre Methode zum ersten Mal vorgestellt hatte. Erst an der zweiten überarbeiteten Auflage von 2018 (vgl. Anm. 16) waren Carrie Friese und Rachel Washburn beteiligt.

19 „Ein Weg zur Repräsentativität nach dem ,postmodern turn'?", auf: Uni Freiburg (Anm. 18). 
anderen untersucht wird; daraus können wiederum "soziale Welten/Arenen als Kartographien der kollektiven Verpflichtungen, Beziehungen und Handlungsschauplätze" ${ }^{\prime 20}$ abstrahiert werden. Auf der höchsten Abstraktionsebene steht schließlich eine "positional map", auf der nicht nur explizit geäußerte, sondern auch unartikulierte und zum Schweigen gebrachte Positionen herausgearbeitet werden können.

„Articulating and Acting Upon Absent Positions Perhaps the most important aspect of doing positional maps is that they push the researcher to see positions not taken in the data, positions that remain unarticulated. Positional maps allow silences to be made to ,speak'. Analyzing, articulating, and discussing silent/silenced positions makes them speak. Noticing what is not present in a situation is notoriously difficult, and the special work these maps do is in helping the researcher to notice such things." ${ }^{21}$

Erste Hinweise auf Elemente kompositorischer Praktiken Wieck/Schumanns finden sich in Quellenbeschreibungen von Textzeugen und Überlieferungsträgern wie der Einführung zur Faksimile-Ausgabe ${ }^{22}$. Aus Wieck/Schumanns Perspektive ergibt sich aus diesen Informationen zunächst diese Situation: Im Hinblick auf einen gemeinsam zu komponierenden Liederzyklus hatte ihr Mann für sie fünf Gedichte aus Friedrich Rückerts Liebesfrühling zur Vertonung ausgewählt. Vier von diesen vertonte sie und schenkte sie ihrem Mann im Juni 1841 zum Geburtstag. Dieser wiederum übermittelte ohne ihr Wissen drei von diesen an den Verlag Breitkopf \& Härtel mit der Bitte, sie neben neun eigenen Vertonungen als gemeinsamen Liederzyklus "Zwölf Gedichte aus Friedrich Rückerts Liebesfrühling" (sein op. 37, ihr op. 12) zu veröffentlichen. Die Drucklegung mit den Vertonungen von Wieck/Schumann an zweiter, vierter und elfter Stelle war für den folgenden September als Geburtstagsüberraschung für sie gedacht. Derart nahm Schumann seiner Frau die Möglichkeit, wichtige Praktiken im Kompositionsprozess selbst auszuführen - nämlich die Vertonungen auf korrekte Notationsweise zu prüfen, ihre letztgültigen kompositorischen Entscheidungen abschließend freizugeben und zu autorisieren sowie mit ihrem Mann die Auswahl und Anordnung ihrer Lieder im Liederzyklus zu diskutieren. Das hier für die Analyse herangezogene Lied "Die gute Nacht, die ich dir sage" WoO 17 ist das vierte des Geburtstagsgeschenks; es wurde weder von Robert Schumann für den Zyklus ausgewählt noch später von Clara Schumann veröffentlicht. ${ }^{23}$ Über die Gründe dafür ist nichts bekannt. Neben dem Widmungsautograph hinterließ die Komponistin - wie oben erwähnt - ein zweites, in dem Arbeitsmanuskripte ${ }^{24}$ nahezu aller ihrer Lieder enthalten sind; das Deckblatt stammt

20 Ebd.

21 Clarke/Friese/Washburn, Situational Analysis, hier S. 172.

22 Clara Schumann, Vier Gedichte von Rückert, Opus 12 und WoO 17, Faksimileausgabe nach dem Autograph des Robert-Schumann-Hauses Zwickau, hrsg. von Thomas Synofzik (= Meisterwerke der Musik im Faksimile, Bd. 42) Laaber 2020. Thomas Synofzik, "Genderspezifische Editionsprobleme? Die Gedichte aus Rückerts Liebesfrühling von Clara Schumann op. 12", in: Louise Farrenc und die Klassik-Rezeption in Frankreich, hrsg. von Rebecca Grotjahn et al. (= Schriftenreihe des Sophie-Drinker-Instituts, Bd. 2), Oldenburg 2006, S. 215-226.

23 Es wurde erst 1992 aus dem Nachlass veröffentlicht (vgl. Clara Schumann, Sämtliche Lieder, Bd. 2: Unveröffentlichte Lieder, hrsg. von Urtext Joachim Draheim et al., Wiesbaden 1992).

24 Ebd., "Revisionsbericht", S. 56. 
von Schumann und ist mit 1842 datiert. Es sind aber auch später entstandene Lieder der Komponistin enthalten. Zur Gesamtsituation, die rund um die künstlerisch-praktische Entstehung der vier Lieder analysiert werden müsste, sind nach Adele Clarke auch einige ökonomische Aspekte heranzuziehen (vgl. Tabelle ${ }^{25}$ ), die dem erweiterten zeitlichen Umfeld jenes Werks zuzurechnen sind, das das erste und einzige gemeinsame der Eheleute bleiben sollte.

25 Elemente einer vollständig zu erfassenden Situation nach Adele Clarke u. a. „SA Ordered Situational Map Template”, <http://study.sagepub.com/sites/default/files/Clarke2e_OrderedTemplate.docx>(20.09.2020).

\author{
Ordered situational map \\ 1. Individual Human Elements/ Actors \\ e.g., key individuals and significant (unorganized) \\ people in the situation, including the researcher \\ 2. Nonhuman Elements/Actants \\ e.g., technologies;material infrastructures; \\ specialized information and/or knowledges; \\ material "things"
}

3. Collective Human Elements/Actors e.g., particular groups; specific organizations

4. Implicated/Silent Actors/Actants

As found in the situation

5. Discursive Constructions of Individual and/or Collective Human Actors

As found in the situation

6. Discursive Construction of Nonhuman Actants

As found in the situation

7. Political/Economic Elements

e.g., the state; particular industry/ies;

local/regional/global orders; political parties; NGOs; politicized issues

8. Sociocultural/Symbolic Elements

e.g., religion; race; sexuality; gender; ethnicity; nationality; logos; icons; other visual and/or aural symbols

9. Temporal Elements

e.g., historical, seasonal, crisis, and/or trajectory aspects

\section{Spatial Elements}

e.g., spaces in the situation; geographical aspects; local, regional, national, and global spatial issues 11. Major Issues/Debates (Usually Contested) As found in the situation; see positional map

12. Related Discourses (Historical, Narrative, and/or Visual)

e.g., normative expectations of actors, actants, and/or other specified elements; moral/ethical elements; mass media and other popular cultural discourses; situation-specific discourses
(Historische) Partizipanden in Praktiken

$>$ Robert Schumann

$>$ Clara Wieck/Schumann

$>$ Analytikerin

$>$ Druckausgabe von Friedrich Rückerts Gedicht ('1836)

$>$ Auswahl/Abschrift von fünf Gedichten durch Schumann D-Zsch 4871/VIII,4

$=5977-\mathrm{A} 3$

$>$,Materialisierungen' der Vertonung

$\rightarrow \quad$ zwei Manuskripte: D-B Mus.ms.autogr. Schumann, K. 5 (6) und D-Zsch 5985-A1

$\rightarrow$ Erstausgabe 1841 mit geteilter Autor*innenschaft

$>$ historische Kompositionslehre und Dichtungstheorie

$>$ Wieck/Schumanns Instrument

$>$ Verlag des Gemeinschaftswerks

$>$ Anonymes Publikum (Käufer*innen von Notendrucken; zahlendes

Konzertpublikum)

$>$ Konzertveranstalter (Dissemination des Gemeinschaftswerks)

$>$ Friedrich Rückert (als ideeller Impulsgeber)

$>$ Friedrich Wieck (als Kritiker des ehelichen, Wirtschaftsbundes')

$>$ Komponist \& Pianistin

$\rightarrow$ Liebespaar

$\rightarrow$ Künstlergemeinschaft

> op. 37| 12 als Symbol für den Erfolg der Liebes-/Künstlergemeinschaft

$>$ Künstler-/Liebesgemeinschaft als geldwerte ,Marke'

> Künstler-/Liebesgemeinschaft als Wirtschaftsgemeinschaft

$>$ Eigentumsrecht in der Ehe (vgl. Preiß 2004)

$>$ Wertschöpfung durch Drucklegung des "Liebesfrühlings"

$>$ Kochbuch als Geschenk Robert Schumanns für Clara Wieck (Weihnachten 1839) und Verweis auf die ihr zugedachte Rolle (vgl. Huber 2014)

> Ehekonsensprozess Clara Wieck vs. Friedrich Wieck (15.6.1839-1.8.1840)

$>$ Injurialklage Robert Schumann vs. Friedrich Wieck (Wiecks Verurteilung April 1841)

$>$ Geschenk an Robert im 9. Monat nach der Eheschließung (12.9.1840)

$\rightarrow$ erste Erfahrungen als Vorsteherin eines Haushalts

$\rightarrow$ erste Erfahrungen mit gemeinsam genutzten Arbeitsmöglichkeiten

$\rightarrow$ 6. oder 7. Monat der ersten Schwangerschaft (Geburt Marie am 1.9.1941)

$>$ Wohnort Leipzig als weniger rentabler Arbeits-/Wirtschaftsstandort als etwa Wien

$>$ Voraussetzungen, wirtschaftliche Durchführbarkeit und tatsächliche Verwirklichung der Künstler-/Liebesgemeinschaft

> Möglichkeiten bzw. Einschränkungen Wieck/Schumanns zu wirtschaftlichen Aspekten beizutragen (von regelmäßigem Üben bis hin zu Konzertreisen)

> Perspektivität/Plots biographischer und musikhistorischer Narrative

$>$ musikhistorisch Forschende als Akteur*innen mit mehr oder weniger normativen Erwartungen 
Insbesondere die Frage nach „Political/Economic Elements, e. g., the state; particular industry/ ies; local/regional/global orders; [...]" zwingt dazu, über die rein künstlerischen Praktiken beim Zustandekommen der Vertonung hinaus zu denken und das gemeinsame Werk und seine Drucklegung sowohl als ökonomischen Wert wie auch als öffentlich symbolische Grundlegung der ,Marke' "wandelndes Liebesgedicht" der ",erhabenen gleichen' Liebenden"26 zu verstehen. Damit erweist sich weniger Friedrich Rückert als Inspirator; es rückt vielmehr Friedrich Wieck als Bezweifler des Schumann'schen Lebensmodells in den Vordergrund - ebenso wie andere Akteur*innen, die sich nicht zu diesen Fragen positioniert hatten (wie etwa Marianne Bargiel als (Schwieger-) Mutter in derselben Zeit) und in den Hintergrund treten. Friedrich Wieck hatte im Ehekonsensprozess u. a. wirtschaftlich argumentiert, um seiner Tochter in ihrer Ehe eine gewisse berufliche Selbstbestimmtheit und ein Mitspracherecht in finanziellen Angelegenheiten zu ermöglichen ${ }^{27}$, und eine Wirtschaftsgemeinschaft seiner Tochter mit Robert Schumann unter anderem dann für fragwürdig gehalten, wenn sie als (potenzielle) Mehrverdienerin, nicht aber entscheidungsbefugte Teilhaberin zum Lebensunterhalt beitragen würde. Die befürchtete Situation trat dann auch tatsächlich ein, als Clara Wieck mittags um 12 Uhr am Tag vor ihrer Volljährigkeit Robert Schumann ehelichte; hätte sie zwölf Stunden gewartet, hätte sie als Volljährige über eben jene Handlungsspielräume verfügen können, die ihr die (letztlich vergebliche) Argumentation des Vaters verschafft hätte. Dass dieser nicht auf Anzeichen - wie etwa die Publikation eines gemeinsamen Werkes - gewartet hätte, dass die Lebensplanung seiner Tochter (anders als er es befürchtet hatte) doch erfolgreich ist und dass das junge Ehepaar nicht auch den Beweis antreten wollen würde, dass alle Einwände des (Schwieger-) Vaters unbegründet waren, ist kaum vorstellbar.

Mit dieser Aufstellung von Elementen möglicher Praktikenketten um Wieck/Schumanns Vertonungen der vier Rückert-Gedichte wäre also erst ein Ausgangspunkt für eine „situational analysis" nach Adele Clarke gelegt. Daran anschließend müssten nun "relational maps" und schließlich eine "positional map" abstrahiert werden. Dies kann im gegebenen Umfang dieses Beitrags nicht geleistet werden, wobei auch fraglich wäre, ob das Ergebnis einer vollständigen Analyse dieser Situation für sich genommen so interessant wäre. Ergiebiger könnte vielmehr ein Vergleich mehrerer ähnlicher Kooperationen anderer "Künstlergatten" sein, für die aber hier ebenfalls nicht der richtige Ort ist. Die Hypothesen jedoch, die durch die Arbeiten an den "relational" und "positional maps" verifiziert werden müssten, lassen erwarten, dass sich etliche unartikulierte Positionen Clara Wieck/Schumanns herausstellen werden - wenn man etwa die zuweilen bemerkenswerte Reaktionslosigkeit Clara Wiecks in ihren Antworten auf einige Briefe

26 Franz Liszt, Gesammelte Schriften, Bd. 4: Aus den Annalen des Fortschritts. Konzert- und kammermusikalische Essays, dt. bearb. von Lina Ramann, Leipzig 1882, hier S. 191.

27 Friederike Preiß, Der Prozeß: Clara und Robert Schumanns Kontroverse mit Friedrich Wieck, Frankfurt a.M. etc. 2004, und dies. "Schumann und Wieck - eine kritische Auseinandersetzung?", in: Übergänge. Zwischen Künsten und Kulturen: Internationaler Kongress zum 150. Todesjahr von Heinrich Heine und Robert Schumann, hrsg. von Henriette Herwig et al., Stuttgart 2007, S. 55-66. 
Schumanns im Hinblick auf die Gestaltung des ehelichen Lebens verfolgt, in denen dieser sich Visionen über die haushälterische Tätigkeit seiner Frau unter Ausschluss der musikinteressierten Öffentlichkeit hingibt ${ }^{28}$ - als ob eine der bestverdienenden Musikerinnen ihrer Zeit es sich nicht leisten könnte, eine Haushälterin zu engagieren. Deutlich wird jedoch, dass die Relationen zwischen der strukturanalytisch zu vermittelnden Schönheit und Eigenart der oben analysierten Vertonung und den übrigen Elementen auf der "situational map" mehr Leerstellen als Verbindungen aufweisen werden. Das vertonte Gedicht kann sich lediglich auf eine Idylle beziehen, während sich die tatsächliche Lebenssituation der Komponistin/Pianistin zwischen realitätsfernen Vorstellungen ihres Mannes und ihrer eigenen sehr realen Kunstausübung noch konsolidieren muss. Die Musikstrukturen der Vertonung bieten - für sich genommen und ohne den vertonten Text - keine verwertbaren Anhaltspunkte, die kartographierte Lücken schließen könnten.

Andere geschichtsbezogene Praxistheorien, die weniger auf qualitative Methoden bauen, betonen, dass "ein praxeologischer Zugang [...] die Perspektive hingegen [erweitert] jedoch nicht nur, indem alle Akteure des Geschehens gleichberechtigt in den Blick rücken und alle verfügbaren Quellen und Repräsentationen herangezogen werden, sondern die Ereignishaftigkeit selbst historisiert wird. Eine so konzipierte Geschichte denkt nicht vom Ergebnis her, sondern sieht dies selbst als Ergebnis von historisch wandelbaren Prozessen der Zuschreibung." ${ }^{29}$

\section{Komponieren als Handeln}

Wenn wir nun schließlich das Sprechen der Pianistin durch/in ihre/n Kompositionen als Handeln betrachten wollen, treten sie selbst als Sinnstiftungszentrum und die Frage nach ihren Adressat*innen in den Fokus:

Wer ist diese Pianistin, die da spricht? Spricht sie als Virtuosin oder als Liedbegleiterin? Macht das einen Unterschied, was ihre kompositorische Praktik angeht, oder nicht? Spricht sie als Frau, als Ehefrau, Mutter oder Tochter? Warum wäre es wichtig, das sagen zu können?

Zu wem spricht sie? Zu ihrem Mann als Widmungsträger in jedem Fall; möglicherweise zum Dichter des vertonten Gedichts durch ihre kompositorische Interaktion mit den Gedichtstrukturen; zum anonymen Publikum oder den Interpret*innen ihrer und folgender Zeiten wahrscheinlich. Zu uns als Analysierende? Ganz bestimmt nicht.

Was sagt sie? Gibt es zusätzlich zum vertonten Gedicht noch einen Subtext, den sie in den musikalischen Strukturen verborgen hat? Diese Frage führt zuweilen zu abenteuerlichen struk-

28 Annegret Huber, „Robert Schumann schenkt Clara Wieck ein Kochbuch. Etüde zur Anwendbarkeit der Kategorie Erinnerungsort beim Close Reading bekannter biographischer Quellen", in: Musik und Erinnern. Festschrift für Cornelia Szabó-Knotik, hrsg. von Christian Glanz et al., Wien 2014, S. 299-308.

29 Marian Füssel, "Praktiken historisieren. Geschichtswissenschaft und Praxistheorie im Dialog”, in: Methoden einer Soziologie der Praxis, hrsg. von Anna Daniel et al., Bielefeld 2015, S. 267-287, hier S. 282. 
turanalytischen Zugriffen, wenn beispielsweise unterstellt wird, die Komponistin und ihr Mann hätten unter Zuhilfenahme einer gewissen motivartigen Tonauswahl Chiffrierungen ${ }^{30}$ vorgenommen, die sogar als Stimmen verstanden werden können, die Spuren sozialer Geschlechter mit sich führten. ${ }^{31}$ Derart wird aber die Komponistin weniger ,zum Sprechen gebracht' als bevormundet; die Analyse wird zum Spurenlesen im Sinne Sybille Krämers, die davon ausgeht, dass 'Spuren' nicht einfach ,da' sind, sondern erst zu Spuren werden, wenn sie als solche behandelt werden: "Spuren entstehen durch die im aktualen Kontext des Spurenlesers verwurzelte und mit der erzählenden Herstellung kausaler Abhängigkeiten kompatible Interpretationsarbeit", denn der "Spurenleser verhält sich als Adressat von etwas, dessen unfreiwilligen Absender er allererst zu rekonstruieren hat" ${ }^{\prime 2}$

\section{B) Die Analysierenden sprechen}

\section{Praktik des Musikanalysierens}

Wenden wir uns nun schließlich dem Musikanalysieren als einer Praktik des Relationen Herstellens zu, die sich bereits im Begriffspaar Analyse-Synthese als Minimal-Definition spiegelt: Musikimmanent werden die zergliederten Musikstrukturen abstrahierend auf einander bezogen; Kontextualisierungen stellen über die Musikanalyse hinausgehend Beziehungen her und können auch zu Praktikenketten führen. Zur Differenzierung der Konstruktion von Relationen bei Kontextanalysen schlägt Tasos Zembylas ${ }^{33}$ vier Möglichkeiten vor; er spricht von einer kausalen Relation, wenn eine Entstehungsbedingung als Ursache für das Phänomen gelten kann - wenn also eine Komponistin nur deshalb in einer bestimmten Weise komponieren würde, weil sie eine Pianistin ist. Bei einer konditionalen Relation wird eine Bedingung zu einer ermöglichenden oder beschränkenden Voraussetzung - wenn etwa jemand zu der Auffassung kommt, dass die Pianistin als Frau Gedichte nicht so gut wie ihr Mann vertonen kann, weil angeblich die Melodie weiblich sei und die Harmonik männlich. ${ }^{34}$ Eine modale Relation bringt eine mögliche, aber nicht hinreichende Rolle für die Entstehung eines Phänomens ins Spiel - wenn etwa eine Ana-

30 Eric Sams, "The Schumann Ciphers", in: The Musical Times 107, H. 1479 (1966), S. 392-393 und S. 395-400. Etwas erschütternd ist zu beobachten, dass den Dechiffrierern nicht auffällt, dass es sich bei dem, was sie als ,Themen' bezeichnen und wie bedeutungstragende Zeichen behandeln, um nichts anderes als gewöhnliche (nicht rhythmisierte und zumeist) Fünftonfolgen einer Tonleiter handelt, ohne die tonale Musik keine solche wäre.

31 Melinda Boyd, "Gendered Voices: The ,Liebesfrühling' Lieder of Robert and Clara Schumann", in: 19th-Century Music 23, H. 2 (1999), S. 145-162. Der in der vorigen Fußnote beschriebene analytische Zugriff wird hier schlicht und unhinterfragt aufgenommen und einer musiksemiotisch unreflektierten Gender-Lesart zugrunde gelegt.

32 Sybille Krämer, Medium, Bote, Übertragung, Kleine Metaphysik der Medialität, Frankfurt a.M. 2008, hier S. 282.

33 Tasos Zembylas, „,Kontext' als wissenschaftliches Paradigma in der Musikforschung?" (unveröffentlichtes Manuskript 2019).

34 Synofzik, Faksimileausgabe, hier S. 11. 
lytikerin davon ausgeht, dass die Komponistin durch ihre Vertonung in einen Dialog mit dem/ der Dichter*in, Widmungsträger*in etc. tritt. Mit einer transitiven Relation wird ein Attribut eines Bereichs auf jedes Phänomen übertragen, das in diesen Bereich eindringt - wenn etwa Musikzeichen ungeachtet ihrer unterschiedlichen semiotischen Qualitäten wie Sprachzeichen behandelt und auf ihre mutmaßlich inhärente Bedeutung untersucht werden (wobei Poststrukturalistinnen annehmen würden, dass nicht einmal Worten unveränderliche Bedeutung innewohnt). Letzterem entgegengesetzt sind Zugriffe, die nicht für sich in Anspruch nehmen, den Musikzeichen Bedeutung zu entnehmen, sondern eingestehen, dass jede Sinnerfüllung von Zeichen mit Bedeutung eine Zuschreibung ist. Mögen auch die Entscheidungen für die eine oder andere Relation mehr oder weniger bewusst getroffen werden, handelt es sich dabei dennoch um Entscheidungen, die zur Analyse als dem Ergebnis einer Praktik geführt haben. Stellt man der Entscheidung für etwas das Vermeiden von etwas anderem gegenüber, kann auch das Unterlassen als Element einer Praktikenkette gefasst werden - wenn etwa die Eignung oder epistemologische Grundlagen eines musikanalytischen Verfahrens vor der Durchführung einer Analyse nicht geprüft werden.

\section{Analysieren als Handeln}

Die scheinbare Unsichtbarkeit des dezentrierten Subjekts der Praxeologie darf nicht mit Neutralität, Wertfreiheit oder gar Objektivität der wissenschaftlichen Praktik verwechselt werden - zumal sich die Situation der heutigen Analysierenden durchaus als Handlung mit Subjekten als Sinnstiftungszentren beschreiben lässt: Die Analysierenden der Gegenwart können in der Regel noch zu ihrer Wissenspraktik befragt werden, sofern sie versäumt haben sollten, ihre Erkenntnisprämissen offenzulegen. Dies wurde spätestens seit 2006 explizit aus dem Bereich der Popularmusikanalyse mit der Forderung nach einem selbstreflexiven "Forschersubjekt" geäußert, welches sich selbst mit seiner epistemischen Wirkung mitten in der Analyse positionieren müsse: „Die radikale Selbstreflexivität des Forschersubjekts muss zudem die Einsicht vermitteln, dass es durch Motive, Ziele und Methoden das klangliche Objekt rahmt und diskursiviert. Dieser Prozess ist expliziter Bestandteil jeder Analyse und gehört daher auch in ihre schriftliche Fassung. ${ }^{36}$ Diese Forderung ist auf jeden musikbezogenen Forschungsbereich zu übertragen - nicht nur den, in dem im engeren Sinn Musikstrukturen analysiert werden.

35 André Doehring, "Probleme, Aufgaben und Ziele der Analyse populärer Musik”, in: Black Box Pop. Analysen populärer Musik, hrsg. von Dietrich Helms et al., Bielefeld 2012, S. 23-42, hier S. 32; siehe auch Mark J. Butler, Unlocking the Groove. Rhythm, Meter, and Musical Design in Electronic Dance Music, Bloomington 2006.

36 Doehring, "Probleme, Aufgaben”, S. 37. 


\section{Praxisformationen um das Musikanalysieren}

Der musikanalytische Diskurs zwischen Praktiken der Musiktheorie, Musikästhetik und Musikhermeneutik kann mit gutem Grund als Praxisformation bezeichnet und könnte auch mit der qualitativen Methode von Adele Clarke analysiert werden. Hier kommt jedoch aus Perspektive der Sozialepistemologie eine weitere Kategorie hinzu - nämlich die Gesamtsituation aller musikanalysierenden Subjekte in Wissensgesellschaften und ihren Institutionen sowie ihre epistemischen Funktionen in individuellen und kollektiven Erkenntnisprozessen. ${ }^{37}$ Bahnbrechend wirkte dahingehend Helen E. Longinos Science as social knowledge. Values and objectivity in scientific inquiry ${ }^{38}$ von 2002 mit ihrer Schlussfolgerung, dass "kognitive Prozesse der Wissenschaft, wie etwa Beobachtung und Schlussfolgerung, an sich soziale Prozesse" - und in der Lesart des vorliegenden Beitrags: in Wissenspraktiken verkörpert - sind. ${ }^{39}$ Dabei wird eine „transzendente aperspektivische Position" der Forschenden abgelehnt, denn "die an der Produktion von Wissen beteiligten Personen sind historisch, geographisch und sozial situiert; ihre Beobachtungen und Überlegungen spiegeln ihre Situation wider" ${ }^{40}$. Wissenschaftlichkeit wird hier aber nicht durch mutmaßliche ,Subjektivität' kompromittiert, denn die Positionen der forschenden Subjekte werden als Merkmal eines Forschungsprozesses in seine Epistemologie eingearbeitet.

\section{Conclusio}

Abschließend stellt sich die Frage nach dem Mehrwert, wenn man das Komponieren und Analysieren von Musik als Praktiken/Praxen/Handlungen mit soziologischen Kategorien zusammendenkt: Dieser entsteht dadurch, dass die epistemische Funktion der musikanalysierenden Subjekte im Erkenntnisprozess problematisiert werden kann, ohne in subjektivistische Nabelschau zu verfallen. Ebenso kann die Funktion der Zeug ${ }^{\star}$ innenschaft ${ }^{41}$ der Analysierenden und die Frage nach der Evidenz von Strukturanalysen problematisiert werden. Damit verbindet sich ein Plädoyer für eine Epistemologie, die danach fragt, was wir überhaupt im Wege des Musikanalysierens wissen können und wie dieses Wissen nicht nur einer ,Illustration' gleich, sondern schlüssig argumentiert in allen Bereichen der Musikforschung kontextualisiert werden kann.

37 Annegret Huber, „Differenz zu Ende denken. Musikstrukturanalytische Wissenspraxen aus poststrukturalistischer Perspektive", in: MUSIK|KULTUR|THEORIE. Festschrift für Marie-Agnes Dittrich, hrsg. von Christian Glanz et al., Wien 2019, S. 227-256.

38 Helen E. Longino, Science as social knowledge. Values and objectivity in scientific inquiry, Princeton 1990.

39 Helen Longino, "The Social Dimensions of Scientific Knowledge", in: The Stanford Encyclopedia of Philosophy, hrsg. von Edward N. Zalta (Summer 2019 Edition), < https://plato.stanford.edu/archives/sum2019/entries/scientific-knowledge-social/> (23.09.2020).Übersetzung A.H.

40 Ebd. Übersetzung A.H.

41 Testimony/Bearing Witness. Epistemology, Ethics, History and Culture, hrsg. Von Sybille Krämer und Sigrid Weigel, London 2017. 


\section{Denn}

„etwas zu historisieren, kann [...] zweierlei bedeuten. Es kann einerseits heißen, dass wir Praktiken unserer eigenen Gegenwart genealogisch in ihrer historischen Gewordenheit betrachten. [...] Andererseits kann Historisierung bedeuten, aus der Anerkennung der historischen Alterität heraus Differenz beobachtbar zu machen. Für den modernen Beobachter merkwürdige, irrationale oder gar befremdliche Vorgänge auf ihren ,praktischen Sinn' (Bourdieu) zu befragen, legt somit erst ihre spezifische Historizität frei." ${ }^{42}$

Zitation: Annegret Huber, „Die Pianistin spricht. Überlegungen zur Epistemologie von Vertonungsanalysen und ihrer Funktion in musikwissenschaftlicher Forschung", in: Die Begleiterin - Clara Schumann, Lied und Liedinterpretation, hrsg. von Rebecca Grotjahn und Nina Jaeschke (= Musikwissenschaft: Aktuelle Perspektiven. Bericht über die Jahrestagung der Gesellschaft für Musikforschung 2019 in Paderborn und Detmold, Bd. 2), Detmold 2020, S. 44-63, DOI: $10.25366 / 2020.83$.

42 Füssel, "Praktiken historisieren”, hier S. 280. 


\section{Abstract}

There is nothing fundamentally wrong with the premise that a pianist like Clara Wieck/ Schumann 'speaks' in her song compositions. This, however, raises a number of epistemological questions that will be discussed in this article. First of all, an explicit distinction is made between the examination of the 'technical' aspects of her compositional practice - in German: Praktik - (which may allow conclusions to be drawn about the pianist's implicit knowledge) on the one hand, and the social aspects of her discursive practice - in German: Praxis - on the other. Thus, it is also necessary to discuss the criteria that the structural-analytical methodology must satisfy, as well as to consider to whom the pianist is actually speaking: to us music researchers of the $21^{\text {st }}$ century? Or should we ask ourselves whether our analysis is not rather a "reading of traces" in the sense of Sybille Krämer, through which we invent the 'producer' of the analyzed 'trace' in the first place? Or to put it another way epistemologically: how do we make the pianist speak? What function does our 'speaking' of her compositions - namely the piano parts in her songs - have in scholarly argumentations?

\section{Kurzvita}

Annegret Huber ( ${ }^{1963}$ in Sindelfingen). Sie studierte Lehramt an Gymnasien an der Hochschule für Musik und Darstellende Kunst Stuttgart, Musikpädagogik an der Musikhochschule Lübeck, Konzertfach Klavier sowie Musiktheorie bei Diether de la Motte an der Hochschule für Musik und darstellende Kunst Wien und Musikwissenschaft an der Universität Wien. Derzeit ist Huber Professorin für Musikwissenschaft (dezidierter Schwerpunkt Analyse der Musik) an der Universität für Musik und darstellende Kunst Wien (Institut für Komposition, Elektroakustik und Tonmeister_innen-Ausbildung). Überdies ist sie Autorin von Bärenreiter Basiswissen Klaviermusik (Kassel 2014) und gibt gemeinsam mit Doris Ingrisch, Therese Kaufmann, Johannes Kretz, Gesine Schröder und Tasos Zembylas Knowing in Performing. Artistic Research in Music and the Performing Arts (im Druck) heraus. 
Die Begleiterin

Clara Schumann

Lied und Liedinterpretation

Herausgegeben von Rebecca Grotjahn und Nina Jaeschke

Musikwissenschaft: Aktuelle Perspektiven 2 
Die Begleiterin 


\section{Musikwissenschaft: Aktuelle Perspektiven}

Bericht über die Jahrestagung der Gesellschaft für Musikforschung 2019 in Paderborn und Detmold

Herausgegeben von Rebecca Grotjahn und Nina Jaeschke

Band 2 


\section{Die Begleiterin \\ Clara Schumann, Lied und Lied- interpretation}

Herausgegeben von Rebecca Grotjahn und Nina Jaeschke

Detmold: Musikwissenschaftliches Seminar der Universität Paderborn und der Hochschule für Musik Detmold 2020 
DOI: $10.25366 / 2020.78$

Online-Version verfügbar unter der Lizenz: Urheberrecht 1.0, $<$ https://rightsstatements.org/page/InC/1.0/?language=de>

Bibliografische Information der Deutschen Nationalbibliothek

Die Deutsche Nationalbibliothek verzeichnet diese Publikation in der Deutschen Nationalbibliografie; detaillierte bibliografische Daten sind im Internet über http://dnb.d-nb.de abrufbar.

\section{Impressum}

Redaktion: Nina Jaeschke, Rebecca Grotjahn und Jonas Spieker Satz: Nina Jaeschke

(C) Musikwissenschaftliches Seminar der Universität Paderborn und der Hochschule für Musik Detmold 2020 


\section{INHALT}

Vorwort $\quad$ VII

Rebecca Grotjahn

Einleitung 1

\section{Beatrix Borchard}

„Frau Klara Schumann der besten Sängerin."

\section{Thomas Synofzik}

"Würde Sie's zu sehr ermüden zu begleiten?" -

Clara Schumann als Lied- und Kammermusikpartnerin

\section{Annegret Huber}

Die Pianistin spricht. Überlegungen zur Epistemologie von Vertonungsanalysen und ihrer Funktion in musikwissenschaftlicher Forschung

\section{Martin Günther}

Liedbegleitung und künstlerische Identität.

Zur Zusammenarbeit Clara Schumanns mit Julius Stockhausen

\section{Ji Young Kim}

Clara Schumann and Jenny Lind in 1850

\section{Kilian Sprau}

"Wozu die Mühe?"

Über Begleiterlizenzen und ihr Schwinden aus der Aufführungspraxis des Kunstlieds. Mit Tonträgeranalysen zu Richard Strauss, "Zueignung” op. 10 Nr. 1 
Gabriele Buschmeier in memoriam 


\section{Vorwort}

Die vorliegenden Bände dokumentieren die Jahrestagung der Gesellschaft für Musikforschung 2019. In den dreieinhalb Tagen vom 23. bis zum 26. September 2019 wurden in Paderborn und Detmold nicht weniger als 185 Beiträge präsentiert, verteilt auf diverse Symposien, Round tables, Freie Sektionen und Postersessions. Sie alle auf einen Nenner bringen zu wollen, ist ein Ding der Unmöglichkeit - und das ist gut so, ist es doch Ziel der Jahrestagungen, die große Vielfalt der Themen und Methoden des Faches Musikwissenschaft abzubilden. Um die thematische Vielfalt der freien Referate angemessen abbilden zu können und gleichzeitig den inhaltlichen Schwerpunkten der beiden hier publizierten Hauptsymposien ausreichend Raum bieten zu können, erscheinen diese in drei Bänden.

„Musikwissenschaft: Aktuelle Perspektiven": Der Titel der kleinen Reihe ist keine Verlegenheitslösung. Musikwissenschaft im Kontext der Digital Humanities; Musikwissenschaft und Feminismus; Musik und Medien; Musikalische Interpretation - schon die Felder, die von den vier Hauptsymposien bespielt wurden, wären noch vor wenigen Jahrzehnten allenfalls an der Peripherie das Faches zu finden gewesen. Sie entsprechen Arbeitsschwerpunkten der Lehrenden am Musikwissenschaftlichen Seminar der Universität Paderborn und der Hochschule für Musik Detmold, das die Tagung ausrichtete. Zugleich nehmen sie Bezug auf aktuelle Ereignisse und Entwicklungen. So erwuchs das von Andreas Münzmay und Joachim Veit organisierte Symposium „Brückenschläge - Informatik und Musikwissenschaft im Dialog" unmittelbar aus den Erfahrungen im Virtuellen Forschungsverbund Edirom (ViFE) und im fakultäten- und hochschulübergreifenden Zentrum Musik-Edition-Medien (ZenMEM). Der 200. Geburtstag von Clara Wieck/Schumann war der Anlass für das von Rebecca Grotjahn geleitete Symposium „Die Begleiterin - Clara Schumann, Lied und Liedinterpretation", das in enger Kooperation mit der Hochschule für Musik Detmold durchgeführt wurde. Das Hauptsymposium „Brückenschläge" wird in einem separaten Band publiziert (Bd. 3 der vorliegenden Reihe). Im Rahmen dieses Symposiums führte die von Stefanie Acquavella-Rauch geleitete Fachgruppe Digitale Musikwissenschaft eine Posterpräsentation durch, die von den Beiträger*innen erfreulicherweise zu kürzeren Texten umgearbeitet wurden, sodass sie hier ebenfalls, zusammen mit den Postern,

publiziert werden können. Hinzu kommen einige Beiträge, die bereits bei der Jahrestagung 2018 in Osnabrück präsentiert wurden. Auch das Hauptsymposium "Die Begleiterin" wird in einem eigenen Band (Bd. 2) publiziert. Die Beiträge zu den beiden anderen Hauptsymposien hingegen werden an anderen Orten veröffentlicht; in Band 1 („Freie Beiträge zur Jahrestagung der Gesellschaft für Musikforschung 2019") der vorliegenden Publikation finden sich jedoch Einführungen und Abstracts. Das Symposium „Komponieren für das Radio" unter Leitung von Antje Tumat und Camilla Bork (Katholieke Universiteit Leuven) behandelte Einflüsse des Mediums auf Kompositionsprozesse sowie durch radiophone Kompositionen bzw. radiophonen Klang ausgelöste Diskurse. Sarah Schauberger und Cornelia Bartsch (Universität Oldenburg) nahmen das 25-jährige Jubiläum der Fachgruppe Frauen- und Genderstudien zum Anlass für einen Generationenaustausch zum Thema "Musikwissenschaft - Feminismus - Kritik": Was wa- 
ren vor einem Vierteljahrhundert Inhalte und Aufgaben einer feministischen Musikwissenschaft und wie kann sich diese heute positionieren?

Bewusst haben wir im Tagungsbericht auf inhaltliche Eingriffe in die Beiträge verzichtet. ${ }^{1}$ Das gilt besonders für die Freien Referate: Es galt, den Charakter der Jahrestagung als Forum für ,freie', d. h. innovative und auch experimentelle Gedanken zu wahren. Einige Kolleg*innen, die die Tagung mit Vorträgen und Posterpräsentationen bereichert hatten, haben sich gegen eine Publikation im vorliegenden Band entschieden - sei es, weil sie eine Möglichkeit fanden, ihre Beiträge in einem inhaltlich passenderen Rahmen zu veröffentlichen, sei es, weil ihre Überlegungen in ihre entstehenden Qualifikationsschriften fließen sollen, oder sei es, weil sie von den Autor*innen in der vorgetragenen Form zunächst verworfen wurden. Auch damit erfüllt eine Freie-Referate-Sektion ihren Zweck: Die Diskussionen mit der versammelten Fach-Öffentlichkeit sollen dabei helfen, Gedanken weiterzuentwickeln und zu verändern. In diesem Sinne sei allen Beteiligten - den Autor*innen, den nichtpublizierenden Referent*innen und den Mit-Diskutant*innen - ganz herzlich gedankt für ihr Mitwirken bei der Tagung.

Unser herzlicher Dank gilt einer Reihe weiterer Personen, die zum Gelingen dieser drei Bände beigetragen haben. Hier ist besonders Jonas Spieker zu nennen, der uns tatkräftig bei der Redaktion geholfen hat. Andrea Hammes (SLUB Dresden) sei herzlich für die Aufnahme unseres Bandes auf musiconn.publish gedankt - wir freuen uns, damit unsererseits zur Etablierung dieser innovativen Publikationsplattform beizutragen.

Erneut möchten wir an dieser Stelle allen Menschen danken, die uns bei der Organisation, Ausrichtung und Finanzierung der Tagung selbst unterstützt haben: der Präsidentin der Universität Paderborn, Prof. Dr. Birgitt Riegraf, dem Rektor der Hochschule für Musik Detmold, Prof. Dr. Thomas Grosse, den Kolleginnen und Kollegen der beiden beteiligten Hochschulen, dem Vorstand der Gesellschaft für Musikforschung, der Universitätsgesellschaft Paderborn und allen Sponsoren. Besonders dankbar sind wir den Mitarbeiter*innen und den studentischen bzw. wissenschaftlichen Hilfskräften des Musikwissenschaftlichen Seminars, die bei der Vorbereitung und Ausrichtung der Tagung immensen Einsatz zeigten - stellvertretend sei an dieser Stelle Johanna Imm erwähnt, die zusammen mit Nina Jaeschke das Herz des Organisationsteams bildete.

Wir widmen diese Reihe Dr. Gabriele Buschmeier, dem langjährigen Vorstandsmitglied der Gesellschaft für Musikforschung, die kurz vor der Publikation dieses Bandes unerwartet verstarb.

Detmold, im September 2020

Rebecca Grotjahn und Nina Jaeschke

Zitation: Rebecca Grotjahn und Nina Jaeschke, "Vorwort”, in: Die Begleiterin - Clara Schumann, Lied und Liedinterpretation, hrsg. von Rebecca Grotjahn und Nina Jaeschke (= Musikwissenschaft: Aktuelle Perspektiven. Bericht über die Jahrestagung der Gesellschaft für Musikforschung 2019 in Paderborn und Detmold, Bd. 2), Detmold 2020, S. VII-VIII, DOI: 10.25366/2020.79.

1 Freigestellt war den Autor*innen auch, ob sie sich für eine gendersensible Sprache entscheiden bzw. welche Form des Genderns sie bevorzugen. 